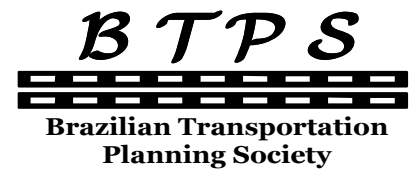

Planning Society
Journal of Transport Literature

Vol. 7, n. 2, pp. 29-51, Apr. 2013

Research Directory
JTL | RELIT

www.transport-literature.org

\title{
Otimização de rede intermodal para o transporte de soja do norte do Mato Grosso ao porto de Santarém
}

[Optimization network for intermodal transport of soybeans in northern Mato Grosso to the port of Santarém]

\author{
Paulo Roberto Grossi Dias Morales, Márcio de Almeida D'Agosto, \\ Cristiane Duarte Ribeiro de Souza* \\ Universidade Federal do Rio de Janeiro (UFRJ), Brazil
}

Submitted 5 May 2012; received in revised form 15 Jul 2012; accepted 17 Jul 2012

\begin{abstract}
Resumo
Este artigo tem como objetivo tem como objetivo propor e estabelecer uma configuração ótima para a rede futura de oferta de transporte prevista pelo Plano Nacional de Logística e Transporte (PNLT) para o horizonte de 2023 para o escoamento de soja do norte do estado do Mato Grosso para o porto de Santarém. Para tanto foi elaborado um procedimento, análogo ao modelo de análise e previsão de demanda de quatro etapas. Foi possível verificar que o Porto de Santarém pode ser considerado uma alternativa para a exportação de soja oriunda do estado do Mato Grosso, visto que a oferta de transporte existente e planejada de Sorriso até o porto de Santarém tem condições de atender a demanda de soja prevista para 2023, e que ainda apresenta um custo de transporte menor do que a alternativa praticada atualmente (utilização dos portos de Paranaguá e Santos), podendo oferecer maior competitividade do produto mato-grossense no mercado mundial.
\end{abstract}

Palavras-Chave: planejamento, transporte de carga, intermodalidade.

\section{Abstract}

The purpose of this paper is to propose and establish an optimal configuration for the future transport network envisaged by the National Plan for Logistics and Transport (PNTL) for the year 2023 for the flow of soybeans from the north of the state of Mato Grosso to the port of Santarém. For this purpose we developed a procedure similar to the four step model for demand analysis and forecasting. It was verified that the port of Santarém can be regarded as an alternative for exporting soy from the state of Mato Grosso, since the existing and planned supply of transportation from Sorriso to the port of Santarém is able to meet the demand for soy scheduled for 2023. It also features a lower transportation cost than the currently practiced alternative (use of the ports of Paranaguá and Santos), being able to offer increased competitiveness for products coming from Mato Grosso in the world market.

Key words: planning, freight transportation, intermodality.

*Email: cristiane@ltc.coppe.ufrj.br.

\section{Recommended Citation}

Morales, P. R. G. D., D'Agosto, M. A. and Souza, C. D. R. (2013) Otimização de rede intermodal para o transporte de soja do norte do Mato Grosso ao porto de Santarém. Journal of Transport Literature, vol. 7, n. 2, pp. 29-51.

- JTL/RELIT is a fully electronic, peer-reviewed, open access, international journal focused on emerging transport markets and published by BPTS - Brazilian Transport Planning Society. Website www.transport-literature.org. ISSN 2238-1031.

This paper is downloadable at www.transport-literature.org/open-access. 


\section{Introdução}

A modernização da agricultura, somada ao processo de globalização mundial e a inserção do estado de Mato Grosso em um cenário estratégico para exportação da produção agrícola, criou a necessidade de elevar a competitividade de seus produtos frente ao mercado internacional.

De acordo com Conab (2012), a região centro-oeste, em 2010, foi responsável por $46 \%$ da produção brasileira de soja. O estado de Mato Grosso foi responsável por 59\% da produção de soja na região centro-oeste e se destaca como o maior produtor de soja do Brasil (27\% da produção brasileira), com ênfase ao norte do estado onde se concentram os municípios que mais produzem o grão. Segundo Fernández (2006), em virtude da convergência de fatores relativos à colonização dirigida e a modernização da agricultura que se desenvolveram ainda nos anos de 1970, o estado de Mato Grosso apresentou um quadro bastante favorável para a expansão das lavouras de soja.

Dentre estes fatores, tem-se à apropriação de tecnologias que permitem utilizar o solo do cerrado para a atividade agrícola em larga escala (monocultivos) e adaptar variedades a fotoperíodo de baixas latitudes (dias longos), propiciando um novo potencial agrícola para região, a construção de infraestrutura (estradas, armazéns e serviços em geral) que possibilita a consolidação de núcleos populacionais, assim como o armazenamento e escoamento da produção e o fluxo migratório das famílias que se deslocaram da região Sul para o estado do Mato Grosso, além de outros estados da Amazônia Legal, buscando oportunidades na atividade agrícola, por meio da aquisição de terras nos programas de colonização pública ou privada, ou, simplesmente, por meio da compra de terceiros (Fernández, 2006).

Em virtude deste potencial de produção de soja conquistado pelo norte do estado de Mato Grosso, verifica-se a necessidade de investimentos em infraestrutura de transportes para que esta produção possa ser escoada até os portos, uma vez que, segundo Barat (1969), "o investimento em transporte é estratégico para uma política de desenvolvimento econômico". A escolha do sistema de transporte a ser utilizado é determinante para o desenvolvimento e crescimento de uma região, pois permite atender mercados mais amplos e distantes de forma mais eficiente e competitiva. 
Neste sentido, os modos mais eficientes para exportação da soja produzida no Brasil (grandes volumes, longas distâncias e valor agregado relativamente baixo) são as ferrovias e hidrovias, que embora exijam um maior tempo de transporte, possuem maior capacidade, podendo trazer economia de custos por ganho de escala e redução de perdas (Hijjar, 2004). O papel do modo rodoviário, por sua vez, seria de atuação como supridor dos modos de maior capacidade, levando os grãos aos terminais ferroviários ou hidroviários.

Com base no que foi apresentado, este trabalho tem como objetivo principal estabelecer a alocação de fluxo ótimo para uma rede futura de oferta de transporte prevista pelo Plano Nacional de Logística e Transporte (PNLT) (MT, 2007) para o horizonte de 2023 para o escoamento de soja do norte do estado do Mato Grosso para o porto de Santarém. Como objetivo adicional, propõe-se um procedimento de estudo, análogo ao modelo de análise e previsão de demanda de transporte de 4 etapas, que configura ferramenta de apoio ao planejamento estratégico do transporte de carga.

Por meio da aplicação deste procedimento verificou-se que o Porto de Santarém pode ser considerado uma alternativa para a exportação de soja oriunda de Mato Grosso, apresentando um custo de transporte menor do que a principal alternativa praticada atualmente (utilização do porto de Santos), podendo oferecer maior competitividade do produto mato-grossense no mercado mundial.

A partir desta introdução, o trabalho se divide em mais 5 itens. O referencial teórico é apresentado no item 1 e o procedimento de estudo é apresentado no item 2, seguido de sua aplicação e apresentação dos resultados (item 3). O item 4 considera a análise dos resultados. Finalmente no item 5 são apresentadas as conclusões, limitações e sugestões de aprimoramentos do estudo.

\section{Referencial teórico}

O problema de alocação de fluxo em uma rede de transporte é normalmente considerado na quarta etapa do modelo de análise e previsão de demanda conhecido como modelo de quatro etapas, componente central do modelo de planejamento de transporte (Souza e D'Agosto, 2013). 
Segundo Morlok (1978), Tedesco (2008) e Magalhães (2008), o modelo de quatro etapas tem inicio com a delimitação da área de estudo, passa pela definição das zonas de tráfego e da rede viária, considera a coleta dos dados, sua calibração e validação. Com isso, pode-se chegar a quatro etapas de previsão de demanda tradicionalmente consideradas pela metodologia: (1) geração de viagens, onde é realizado um levantamento de variáveis que serão usadas para estimar o número total de produção e atração de viagens em cada zona da área em estudo; (2) distribuição de viagens, onde as viagens obtidas são distribuídas entre as zonas de tráfego, produzindo uma matriz de viagens também chamada de matriz origemdestino; (3) divisão modal, onde as viagens da matriz origem-destino são divididas entre os diferentes modos de transportes existentes, gerando matrizes origem-destino por modo de transporte e (4) alocação de fluxo, onde as viagens de cada modo correspondente são alocadas à rede viária existente ou em planejamento. A escolha das rotas pode estar associada a um conjunto de fatores variando inclusive em função do equipamento a ser usado, porém, alguns fatores de decisão como rapidez, retidão, pouco congestionamento e menor caminho são comuns.

Os métodos mais comumente utilizados na etapa de alocação de fluxos são: método "tudo ou nada", método STOCH, método incremental, método de restrição de capacidade, método de equilíbrio do usuário, método de equilíbrio do usuário estocástico e método do sistema ótimo Pereira (2007). A seguir apresenta-se uma descrição sucinta destes métodos com base em Caliper (1996) e Lemes (2005).

No método tudo ou nada, aloca-se todo o tráfego entre os pares de O/D pelo menor caminho, desconsiderando a capacidade e o nível de congestionamentos das ligações. Este método também não considera que os tempos de viagem são dependentes do fluxo de tráfego no arco, tomando-os como um parâmetro fixo.

Já o método STOCH (Estoque) busca distribuir o fluxo entre os diferentes caminhos existentes entre os pares $\mathrm{O} / \mathrm{D}$, de modo que a proporção do fluxo nas diferentes ligações é igual à probabilidade de escolha de cada um deles. Este método não tende a um equilíbrio, pois desconsidera o volume de tráfego.

No método incremental frações do tráfego são alocadas em passos, correspondentes a uma proporção fixa da demanda total. Após cada passo, os tempos de viagem nas ligações são 
recalculados com base nos volumes em cada um deles. Este modelo não permite uma solução de equilíbrio e seus resultados são influenciados pela ordem na qual os volumes dos pares de O/D são alocados.

O método de restrição de capacidade se aproxima de uma solução de equilíbrio, pela iteração entre o método "tudo ou nada" e o cálculo dos tempos de viagem derivados da função de congestionamento dos arcos baseada na capacidade das ligações. Este método não converge para uma solução de equilíbrio e seus resultados são sensíveis a quantidade de iterações realizadas.

No método de equilíbrio realiza-se um processo iterativo que busca alcançar uma solução convergente, na qual os tempos de viajem não podem mais ser melhorados com a troca de rotas. Em cada iteração, os fluxos nos arcos são calculados, incorporando os efeitos da restrição de capacidade e a interdependência destes com o tempo de viagem. Assume-se que o viajante conhece os atributos das alternativas da rede e as escolhas das rotas que minimizam o tempo ou custo de viagem, e que todos os viajantes têm as mesmas estimativas dos atributos da rede.

O método de equilíbrio do usuário estocástico é uma generalização do método do equilíbrio, porém mais realista, pois assume que os viajantes não têm todas as informações sobre os atributos da rede e de seus custos de viagens em diferentes sentidos. Método consistente com o conceito de aplicação de modelos de escolha discreta para escolha da rota.

O método do sistema ótimo minimiza o tempo total de viagem na rede, indicando aos usuários quais rotas eles devem usar, reduzindo assim, o congestionamento da rede. Apesar de difícil aplicação em casos reais, é muito usado na análise de cenários de ITS (Intelligent Transportation System).

Com o objetivo de melhor distribuir os fluxos de tráfego em uma rede de transporte, pode-se utilizar algoritmos de fluxo de custo mínimo, como o algoritmo "Out of Kilter" e o algoritmo de Busacker e Growen, este último de fácil implementação e bom desempenho computacional (Campos, 1997). Como será detalhado no próximo item, o algoritmo de Busacker e Growen pode ser empregado sobre o método de restrição de capacidade. 


\section{Procedimento de estudo}

O procedimento de estudo, que guarda alguma analogia ao modelo de análise e previsão de demanda de quatro etapas, como pode ser visto na Figura 1, é composto por 4 fases que permitem seu desenvolvimento de forma sequencial e estruturada: (1) Identificação do vetor logístico a ser considerado, (2) Análise da oferta de transporte para a região de estudo, (3) Análise da demanda de soja na região de estudo e (4) Alocação de fluxo na rede de transporte.

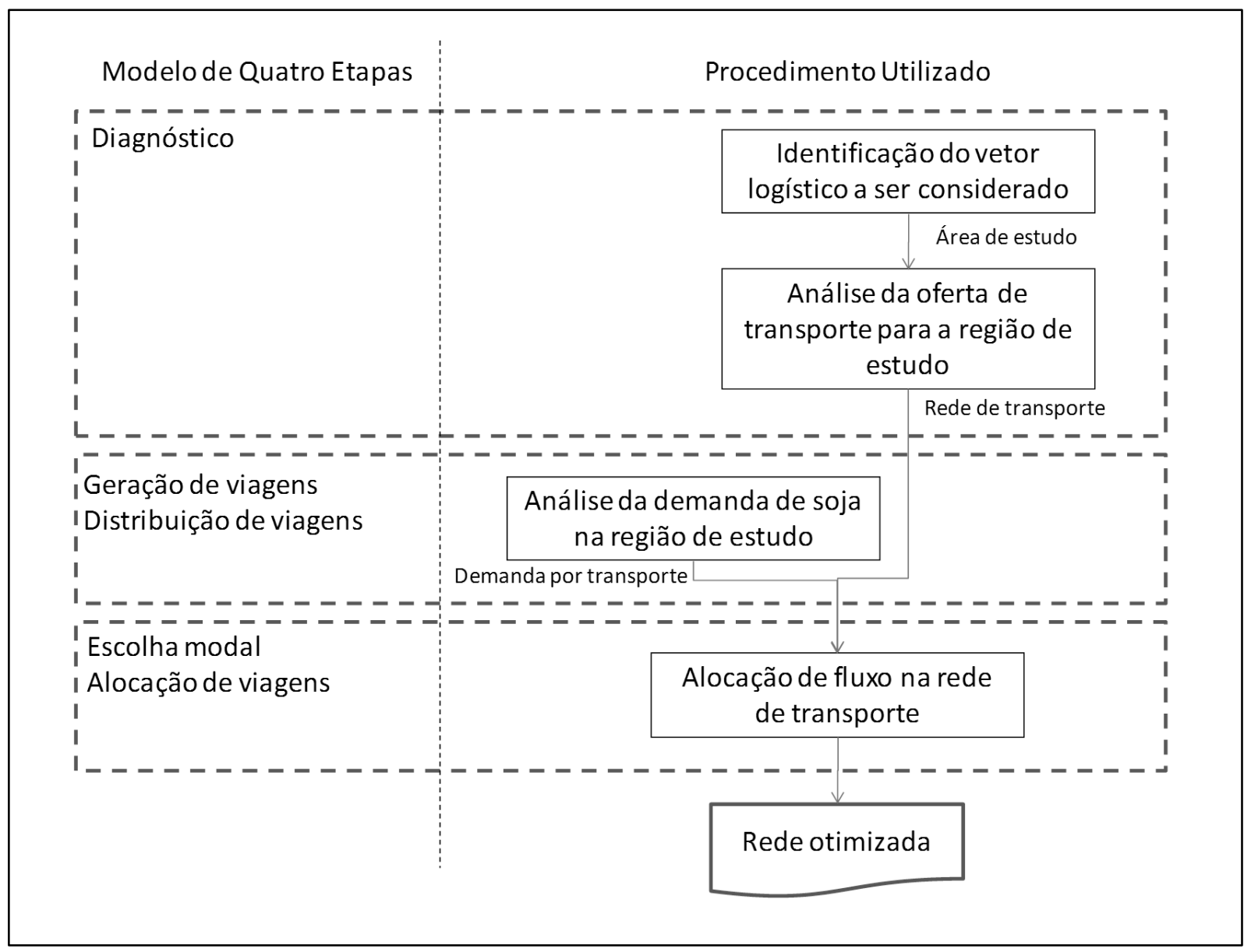

Figura 1 - Procedimento utilizado e sua analogia com o modelo de quatro etapas ${ }^{1}$

Para alocação do fluxo na rede de transporte, a ferramenta de otimização utilizada foi o algoritmo de fluxo a custo mínimo de Busacker e Gowen, por meio do pacote de recursos do software Transportation Planning Sofware - Transcad 5.0 da Caliper Corporation, considerando o custo operacional como parâmetro principal de calibração dos arcos representados pelas vias de escoamento e terminais de transbordo, e as restrições de

\footnotetext{
${ }^{1}$ Fonte: elaboração própria.
} 
capacidade para cada segmento como parâmetro adicional. A utilização do algoritmo de fluxo a custo mínimo e do software Transcad 5.0 possibilitam, em função do limitado tamanho da rede em estudo, condensar as etapas de geração de viagens e distribuição de viagens do modelo de quatro etapas, em uma etapa denominada análise da demanda de soja na região de estudo. Da mesma forma, as etapas escolha modal e alocação de fluxo foram sintetizadas na etapa alocação de fluxo na rede de transporte, conforme procedimento proposto (Figura 1).

Dada uma rede com nós e arcos, os nós para aplicação do algoritmo, foram transformados em arcos fictícios e representam os terminais de transbordo e portuários para transferência de carga entre os modos de transportes analisados. Os arcos representam os trechos percorridos entre nós em cada modo de transporte considerado. As restrições de capacidade máxima de transporte de carga foram inseridas apenas nos arcos. O software Transcad 5.0 foi utilizado para o processamento do algoritmo e para a demonstração dos resultados.

O algoritmo de Busacker e Gowen é um procedimento iterativo, que busca alocar uma quantidade "q" de fluxo com o menor custo possível $\left(\mathrm{d}_{\min }\right)$ utilizando, a cada iteração, um algoritmo de caminho mínimo para definir o caminho de custo mínimo onde é alocado o máximo de fluxo $\left(f_{\max }\right)$ possível respeitando as restrições de capacidade de cada $\operatorname{arco}\left(\mathrm{C}_{\mathrm{ij}}\right)$. Posteriormente, é necessário utilizar um procedimento de modificação da rede para definir um novo caminho mínimo onde será alocada a nova quantidade de fluxo máximo possível, e assim sucessivas vezes, até que se tenha atingido o fluxo "q" desejado ou até que não se encontre nenhum caminho para se alocar o fluxo que ainda não foi alocado (Busacker e Gowen, 1961).

O problema do fluxo a custo mínimo, de acordo com Morales (1993), pode ser determinado considerando o problema de enviar uma determinada quantidade de valor "q" (que se denominará fluxo alvo) de uma origem $\mathrm{S}$ para um destino $\mathrm{T}$ em uma rede $\mathrm{G}$ na qual cada arco ( $a, b)$ tem capacidade $C_{i j}$, bem como custo positivo $d_{i, j}$ associado a ele. No caso da rede em estudo, o custo di,j representa os custos operacionais anteriormente calculados. Portanto se "q" for maior do que o valor do fluxo máximo $\left(\mathrm{f}_{\max }\right)$, não existirá solução viável. Porém, se o valor de "q" for menor ou igual a $f_{\max }$, haverá diversos fluxos alocados nos arcos, tornando o problema viável. O problema de fluxo a custo mínimo pode ser formalmente apresentado pela modelagem apresentada pelas Equações de (1) a (5). 


$$
\operatorname{Minimizar} \sum_{i j} d_{i j} * f_{i j}
$$

Sendo $\mathrm{f}_{\mathrm{ij}} \mathrm{o}$ fluxo enviado do nó i para o nó j, sujeito às seguintes restrições:

1. Para todo o $\operatorname{arco}(i, j)$ em $G$;

$$
0 \leq \mathrm{f}_{\mathrm{ij}} \leq \mathrm{C}_{\mathrm{ij}}
$$

2. Para o nó origem s em G tem-se;

$$
\sum_{i} f_{s i}-\sum_{i} f_{i s}=B
$$

Onde $\sum_{i} f_{i j}$ são todos os arcos que chegam ao nó j e $\sum_{j} f_{i j}$ são todos os arcos que saem do nó i.

3. Para o nó destino t em $\mathrm{G}$ tem-se:

$$
\sum_{i} f_{t i}-\sum_{i} f_{i t}=-B
$$

4. Para cada um dos nós restantes, denominado nós intermediários tem-se:

$$
\sum_{i} f_{j i}-\sum_{i} f_{i j}=0
$$

Observar que se todo $\mathrm{C}_{\mathrm{ij}}=1 \mathrm{e} \mathrm{b}=1$, isto é, se não houver nenhuma restrição de capacidade de arcos, o problema é reduzido a achar o caminho mínimo de s para $t$ com $\mathrm{d}_{\mathrm{ij}}$ como o custo (distância ou peso).

Sempre que enviar uma quantidade fluxo através de uma rede em que haja restrição de capacidade de arco imposta, existirá um caminho mínimo se a menor capacidade de arco deste caminho não for menor que "q". Por outro lado, se o valor do fluxo alvo "q" é maior do que a menor capacidade de arco ao longo do caminho mínimo, o restante do fluxo terá que ser enviado através do próximo caminho de menor custo. 


\section{Aplicação do procedimento de estudo}

A aplicação do procedimento de estudo teve o objetivo de estabelecer uma configuração ótima para a rede futura de oferta de transporte prevista pelo Plano Nacional de Logística e Transporte (PNLT) (MT, 2007) para o horizonte de 2023 para o escoamento de soja do norte do estado do Mato Grosso para o porto de Santarém.

\subsection{Identificação do vetor logístico a ser considerado}

"Vetores logísticos" são espaços territoriais brasileiros onde há uma dinâmica socioeconômica que apresenta certa homogeneidade dos arranjos produtivos, dos deslocamentos preponderantes nos acessos a mercados internos e a exportação, dos interesses comuns da sociedade, dos patamares de capacidades tecnológicas e gerenciais e dos problemas e restrições comuns, que podem convergir para a construção de um esforço conjunto de superação de entraves e desafios (MT, 2007).

Embora esses espaços possam conter heterogeneidades internas, eles representam uma repartição do território nacional sobre o qual suas potencialidades podem ser desenvolvidas independentes das fronteiras regionais, estaduais ou municipais, tendo o transporte um papel preponderante na estruturação física desse território do entorno. Seguindo esta lógica, foram estabelecidos pelo PNLT sete vetores logísticos principais - Amazônico, Centro-Norte, Nordeste Setentrional, Nordeste Meridional, Leste, Centro-Sudeste e Sul.

O Vetor Logístico Amazônico será a referência desse estudo, considerando que nesse vetor se enquadram todas as ofertas de transportes da rede em análise para o horizonte de 2023 do PNLT, assim como a principal região demandante de soja dessa pesquisa.

\subsection{Análise da oferta de transporte para a região de estudo}

Em 2023, há a previsão de deslocamento do fluxo de escoamento da soja para o norte com ênfase no uso da intermodalidade, por permitir a redução dos custos de transporte (MT, 2007 e Fajardo, 2006). Estima-se que o frete total para a soja escoada para o porto de Santarém (PA) seja menor em relação ao praticado para o portos de Santos (SP), principal porto 
(76,5\%) utilizado para o escoamento de soja oriunda do município de Sorriso-MT (Lavorentte, 2011).

De acordo com Marrano (2009) baseado em estudos realizados pelo Comitê da BR-163, é possível verificar que, em virtude do porto de Santarém estar localizado mais próximo, cerca de cinco mil milhas náuticas, da Europa do que o Porto de Santos, o custo do escoamento da produção de soja pelo porto de Santarém pode ser de $20 \%$ a $30 \%$ menor. A redução no custo do transporte pode proporcionar maior competitividade do produto mato-grossense no mercado mundial.

As vias analisadas para o escoamento de soja da região demandante para o porto de Santarém são as seguintes:

\subsubsection{Rodovia BR-163}

A BR-163 é a rodovia federal que integra o Centro-Norte ao Centro-Oeste e Sul do Brasil. Esta rodovia está pavimentada do sul para o norte até Guarantã do Norte (MT) a 702 km de Cuiabá (MT), seguindo $1.152 \mathrm{~km}$ em direção a Santarém (PA) por uma estrada não pavimentada (DNIT, 2010). O percurso total da Cuiabá-Santarém a ser asfaltado integra o Plano de Desenvolvimento Sustentável para a Área de Influência da BR-163, que engloba 65 municípios do Pará, 35 de Mato Grosso e 5 do Amazonas. A área que compreende essas cidades totaliza 974 mil metros quadrados.

A pavimentação dessa rodovia federal até o porto de Santarém vem sendo esperada pelos produtores de grãos das regiões do médio-norte e norte de Mato Grosso há muito tempo. Com o acesso até o porto totalmente pavimentado, o produtor de Mato Grosso ganhará maior competitividade, já que o corredor permitirá que o valor do frete seja reduzido em até 30 dólares por tonelada. Esta economia vale tanto para a exportação da produção local, como para quem busca insumos importados de outros países para o campo. 


\subsubsection{Hidrovia Tapajós/Teles-Pires}

A hidrovia Tapajós/Teles-Pires favorece o escoamento da produção agrícola de Mato Grosso por possibilitar a ligação das regiões norte e nordeste de Mato Grosso e centro-sul do Pará. A hidrovia do Tapajós/Teles-Pires pode ser considerada uma opção de implementação do comércio exterior, com sensíveis reflexos para geração de empregos e surgimento de novos empreendimentos.

O rio Tapajós, afluente da margem direita do rio Amazonas, tem $851 \mathrm{~km}$ de extensão até a confluência dos rios Teles-Pires e Juruena (AHIMOR, 2012). A hidrovia Tapajós/Teles-Pires, pode se tornar um elemento fundamental na consolidação da infraestrutura de transporte para tornar o Mato Grosso e parte da região Centro-Oeste em "celeiros mundiais" da produção de grãos. Podendo ainda, proporcionar inúmeros benefícios regionais. O prazo de execução das obras necessárias à consolidação da via navegável e implantação da navegação comercial é de cerca de 3 anos.

\subsubsection{Ferronorte (Ramal Sorriso-Santarém)}

A Ferronorte e o seu ramal entre os municípios de Sorriso e Santarém não foi indicada para investimentos pelo PNLT por questões ambientais. No entanto, esta se apresenta como uma alternativa possível para o futuro, pois ligará Sorriso (maior produtor de soja no Brasil) à Santarém, promovendo a integração dos modos existentes, com redução significativa dos custos operacionais e por consequência das tarifas (CP Empreendimentos, 2007).

\subsection{Análise da demanda de soja na região de estudo}

O PLNT utilizou o modelo EFES - Economic Forecasting Equilibrium System, desenvolvido pela FEA/USP, através da FIPE (Fundação Instituto de Pesquisas Econômicas), para a calibração da taxa de crescimento da produção e consumo da soja que resultou na montagem da matriz de "déficits" e "superávits" (relações de produção e consumo) entre as microrregiões homogêneas, de acordo com a Tabela 1. 
Tabela 1 - Matriz de "déficits" e "superávits"2

\begin{tabular}{lcc}
\hline \multicolumn{3}{c}{ Produto: Soja / Ano: 2023 / Medida: [T X $\left.10^{3}\right]$} \\
\hline Item & Brasil & Mato Grosso \\
\hline Produção liquida & $117.108,3$ & $32.604,3$ \\
Importação & 0,0 & 0,0 \\
Recebeu de outros estados & $70.852,2$ & $12.627,4$ \\
Total da oferta & $187.960,5$ & $45.231,7$ \\
Consumo & $70.852,2$ & $12.627,4$ \\
Exportação & $46.256,1$ & $15.327,0$ \\
Expediu para outros estados & $70.852,2$ & $17.276,8$ \\
Total da demanda & $187.960,5$ & $45.231,7$ \\
\hline
\end{tabular}

Verifica-se que, no ano de 2023, a região do Mato Grosso não precisará mais importar soja e que 15.327 milhões de toneladas de soja por ano serão destinadas para exportação, caracterizando a demanda total que será alocada na rede de transporte em estudo.

O município de Sorriso é o maior produtor de soja do estado de Mato Grosso (Conab, 2012) e está localizado estrategicamente em sua área central. Por essas razões, foi considerado como a área de influência deste estudo e como centroide para o escoamento de toda a produção do norte do Mato Grosso para o porto de Santarém (PA).

\footnotetext{
${ }^{2}$ Fonte: adaptado de MT (2007).
} 


\subsection{Identificação, representação e alocação de fluxos na rede de transporte}

Serão considerados como parâmetros de alocação para a otimização da rede em análise o custo operacional das vias, considerando as restrições de capacidade das vias e terminais de transbordo para o atendimento da demanda pelos os diferentes modos de transportes conforme investimentos previstos no PNLT para o horizonte 2023.

\subsubsection{Topologia da rede em análise}

A concepção da rede de transporte levou em consideração a realidade física das vias e terminais existentes e/ou previstos, ligando um nó de origem no município de Sorriso a um nó de destino no porto de Santarém (PA). Trata-se de uma rede ponderada, composta por nós e arcos, em que as variáveis de otimização consideradas em cada arco foram a capacidade e o custo operacional correspondentes a cada um deles. Em função da ferramenta utilizada (software Transcad 5.0), foram criados arcos fictícios para a representação dos terminais de transbordo e terminais portuários, onde ocorrem transferências de cargas intramodal ou intermodal. A Figura 2 representa a topologia da rede em análise. A Tabela 2 identifica todos os arcos utilizados nesta representação.

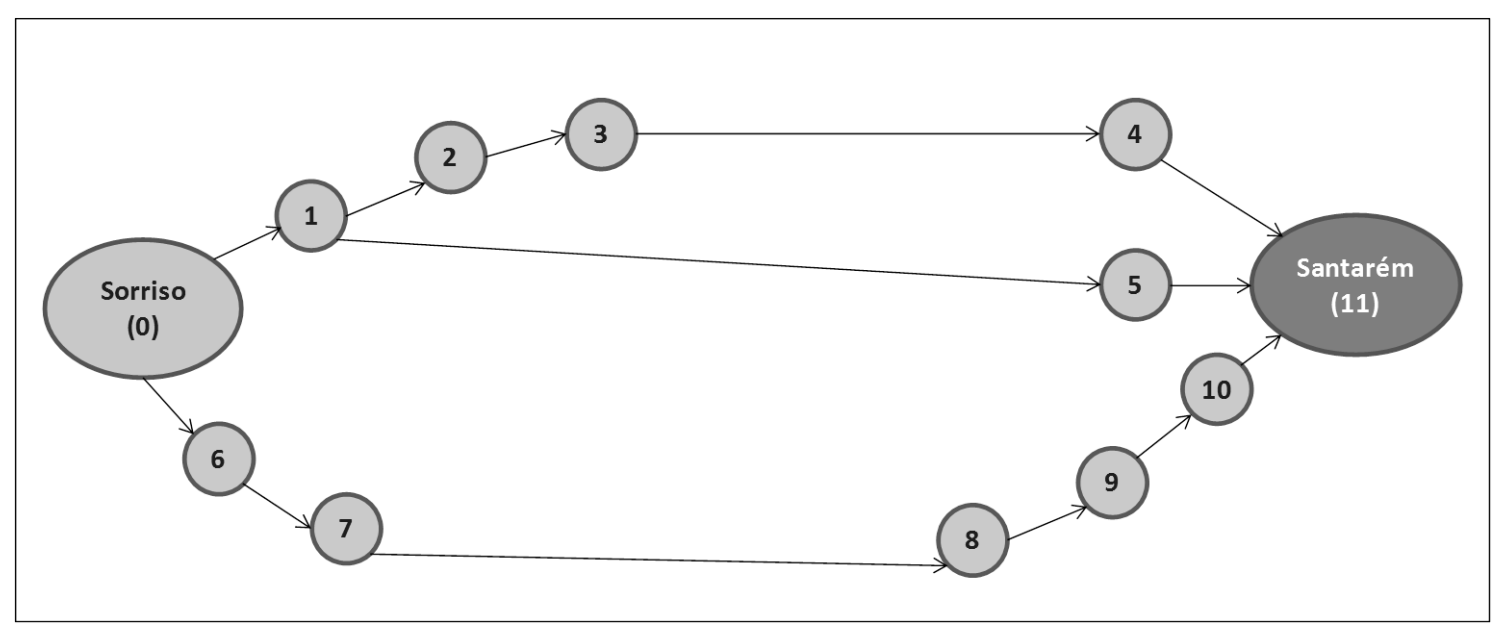

Figura 2 - Esquema da rede em análise ${ }^{3}$

\footnotetext{
${ }^{3}$ Fonte: elaboração própria.
} 


\section{Tabela 2 - Identificação dos arcos da rede ${ }^{4}$}

\begin{tabular}{|c|c|}
\hline Arcos da rede & Trechos correspondentes aos arcos da rede \\
\hline $\operatorname{Arco}(0,1)$ & Acesso rodoviário de Sorriso até a BR-163 \\
\hline $\operatorname{Arco}(0,6)$ & $\begin{array}{l}\text { Acesso rodoviário de Sorriso ao terminal de transbordo rodo- } \\
\text { ferroviário da Ferronorte (ramal Sorriso-Santarém) }\end{array}$ \\
\hline $\operatorname{Arco}(6,7)$ & $\begin{array}{l}\text { Arco fictício representando o terminal de transbordo rodo-ferroviário } \\
\text { da Ferronorte (ramal Sorriso-Santarém) }\end{array}$ \\
\hline $\operatorname{Arco}(1,2)$ & $\begin{array}{l}\text { Trecho rodoviário da BR-163 até o terminal de transbordo rodo- } \\
\text { hidroviário da hidrovia Teles-Pires/Tapajos }\end{array}$ \\
\hline $\operatorname{Arco}(2,3)$ & $\begin{array}{l}\text { Arco fictício representando o terminal de transbordo rodo-hidroviário } \\
\text { entre a rodovia BR-163 e a hidrovia Tapajós/Teles-Pires }\end{array}$ \\
\hline $\operatorname{Arco}(1,5)$ & $\begin{array}{l}\text { Trecho rodoviário da BR-163 até o terminal de transbordo rodo- } \\
\text { hidroviário do porto de Santarém }\end{array}$ \\
\hline $\operatorname{Arco}(3,4)$ & $\begin{array}{l}\text { Trecho hidroviário da hidrovia Tapajós/Teles-Pires até o terminal de } \\
\text { transbordo do porto de Santarém }\end{array}$ \\
\hline $\operatorname{Arco}(4,11)$ & $\begin{array}{l}\text { Arco fictício representando o terminal de transbordo do porto da } \\
\text { hidrovia Tapajós/Teles-Pires para o porto de Santarém }\end{array}$ \\
\hline $\operatorname{Arco}(5,11)$ & $\begin{array}{l}\text { Arco fictício representando o terminal de transbordo rodo-hidroviário } \\
\text { da BR-163 para o porto de Santarém }\end{array}$ \\
\hline $\operatorname{Arco}(7,8)$ & $\begin{array}{l}\text { Trecho da Ferronorte ligando a região de Sorriso ao terminal de } \\
\text { transbordo ferro-rodoviário da Ferronorte no porto de Santarém. }\end{array}$ \\
\hline $\operatorname{Arco}(8,9)$ & $\begin{array}{l}\text { Arco fictício representando o terminal rodo-ferroviário da Ferronorte } \\
\text { para o terminal porto de Santarém }\end{array}$ \\
\hline $\operatorname{Arco}(9,10)$ & $\begin{array}{l}\text { Acesso rodoviário ligando o terminal de transbordo ferro-rodoviário } \\
\text { da Ferronorte para o terminal do porto de Santarém. }\end{array}$ \\
\hline $\operatorname{Arco}(10,11)$ & $\begin{array}{l}\text { Arco fictício representando o terminal de transbordo rodo-hidroviário } \\
\text { do porto de Santarém de acesso pela Ferronorte. }\end{array}$ \\
\hline
\end{tabular}

\footnotetext{
${ }^{4}$ Fonte: elaboração própria.
} 


\subsubsection{Alocação de fluxos na rede de transporte}

Após a especificação da rede de transporte intermodal, é necessária a identificação dos valores de capacidade e custos que devem ser alocados em cada um dos arcos em análise, constituindo-se parâmetros estruturantes e definidores dos fluxos que serão alocados dentro de um critério de otimização que visa minimizar custos operacionais, até o limite do valor total da demanda definido anteriormente.

\subsubsection{Custos e capacidades alocados na rede}

Os arcos que fazem parte da rede compreendem os 3 principais modos de transportes considerados nesta análise, estando apenas a BR-163 mais avançada em termos de especificação de projeto, atendimento às questões ambientais e obras. As capacidades projetadas das vias e terminais para escoamento de soja foram estimadas de acordo com os dados disponíveis no PNLT (MT, 2007).

a. Capacidade projetada das vias e terminais

Nesse artigo não foi considerada qualquer limitação de capacidade nos terminais de transbordo e portuários, uma vez que o enfoque é dado aos parâmetros da rede intermodal de transportes, considerando a limitação de capacidade nos arcos da rede que representam os modos de transporte, bem como o custo operacional para cada segmento em que o fluxo foi alocado.

Estima-se a capacidade de carga do ramal Sorriso-Santarém da Ferronorte como de 20 milhões de t/ano de soja (CP Empreendimentos, 2007) análogo ao do trecho ligando CuiabáSantarém e admitido como válido para o ramal Sorriso-Santarém. Para a estimativa da capacidade de carga da BR-163 após sua pavimentação, foi considerado o EVTE (Estudo de Viabilidade Técnica-Econômica), elaborado por DNIT/IME (2005), que definiu uma capacidade projetada de aproximadamente 15 milhões de t/ano, a partir de dados primários de pesquisa de campo (volumétrica e classificatória) que definiu o tráfego atual e futuro desta rodovia até o ano de 2032. Para o caso da hidrovia Tapajós/Teles-Pires, a capacidade projetada de 12 milhões de t/ano foi obtida por Fialho (2010). 


\section{b. Custos operacionais}

De acordo com Branco e Martins (2006), existe uma relação de, aproximadamente, 1:2:3 entre os custos operacionais dos modos envolvidos. Sendo assim, admite-se o custo operacional de 34 dólares $/ \mathrm{t}-\mathrm{km}^{*} 10^{3}$ para o modo rodoviário; de 21 dólares/t-km*10 ${ }^{3}$ para o modo ferroviário e de 12 dólares/ $\mathrm{t}-\mathrm{km}^{*} 10^{3}$ para o modo hidroviário. Com base no valor médio do dólar em maio de 2010 esses valores foram convertidos para a unidade monetária nacional, chegando aos valores de custo operacional $[\mathrm{R} \$ / \mathrm{t} . \mathrm{km}]$ apresentados na Tabela 3.

c. Custos nos terminais de transbordo, terminais portuários e acessos

Para fins deste artigo, considerou-se para os terminais de transbordo e terminais portuários, o valor de 1 unidade monetária $[\mathrm{R} \$ \mathrm{t}]$, conforme ilustrado na Tabela 3 , pois o enfoque da otimização da rede analisada é a competitividade entre as vias intermodais. Nos acessos rodoviários, foram considerados os mesmos custos operacionais adotados para a BR-163 e apresentados na Tabela 3 .

Tabela 3 - Custos totais que serão alocados na rede ${ }^{5}$

\begin{tabular}{lcc}
\hline \multicolumn{1}{c}{ Vias analisadas na rede } & $\begin{array}{c}\text { Custos } \\
\text { operacionais } \\
\text { alocados aos } \\
\text { arcos [R\$/t.km] }\end{array}$ & $\begin{array}{c}\text { Custos nos terminais de } \\
\text { transbordo } \\
\text { (transferência de carga), } \\
\text { terminais portuários e } \\
\text { acessos [R\$/t] }\end{array}$ \\
\hline BR-163 & 0,063 & 1 \\
Hidrovia Tapajos/Teles-Pires & 0,022 & 1 \\
$\begin{array}{l}\text { Ferronorte - Ramal projetado Sorriso- } \\
\text { Santarém }\end{array}$ & 0,039 & 1 \\
\hline
\end{tabular}

\footnotetext{
${ }^{5}$ Fonte: elaboração própria.
} 


\subsection{Resultados}

Para a demonstração de resultados, foram confrontadas as alocações de fluxos resultantes das ponderações dos arcos da rede com suas respectivas restrições de capacidade, de modo a identificar quais das vias consideradas no estudo são economicamente mais viáveis sob o ponto do poder público, pois é este o provedor de infraestrutura de transporte no longo prazo. Em menor escala, este enfoque deveria convergir com aquele praticado pelo embarcador.

A Tabela 4 representa todas as ponderações que serão consideradas em cada arco da rede com suas respectivas alocações de fluxos. Estando, na Figura 3, os resultados obtidos da otimização da rede por meio do uso do algoritmo Busacker e Gowen e uso do software Transcad 5.0.

Tabela 4 - Ponderações utilizadas nos arcos para o cenário 1 e $2^{6}$

\begin{tabular}{|c|c|c|c|c|}
\hline $\operatorname{Arcos}(\mathbf{i}, \mathbf{j})$ & $\begin{array}{c}\text { Custo } \\
\text { total } \\
{[\mathbf{R} \$ / \mathbf{t} . \mathbf{k m}]}\end{array}$ & $\begin{array}{c}\text { Custo de } \\
\text { transferência } \\
{[R \$ / t]}\end{array}$ & $\begin{array}{c}\text { Capacidade } \\
\text { projetada das vias } \\
\text { [t/ano] }\end{array}$ & $\begin{array}{c}\text { Fluxo alocado } \\
{[\mathbf{t}]}\end{array}$ \\
\hline $\operatorname{Arco}(0,1)$ & 0,063 & $X$ & 15000000 & 12.000000 \\
\hline $\operatorname{Arco}(0,6)$ & 0,063 & $\mathrm{X}$ & 15000000 & 3.327000 \\
\hline $\operatorname{Arco}(6,7)$ & $\mathrm{X}$ & 1 & Infinita & 3.327000 \\
\hline $\operatorname{Arco}(1,2)$ & 0,063 & $\mathrm{X}$ & 15000000 & 12.000000 \\
\hline $\operatorname{Arco}(2,3)$ & $X$ & 1 & Infinita & 12.000000 \\
\hline $\operatorname{Arco}(1,5)$ & 0,063 & $\mathrm{X}$ & 15000000 & 0 \\
\hline $\operatorname{Arco}(3,4)$ & 0,022 & $\mathrm{X}$ & 12000000 & 12.000000 \\
\hline $\operatorname{Arco}(4,11)$ & $\mathrm{X}$ & 1 & Infinita & 12.000000 \\
\hline $\operatorname{Arco}(5,11)$ & $\mathrm{X}$ & 1 & Infinita & 0 \\
\hline $\operatorname{Arco}(7,8)$ & 0,039 & $\mathrm{X}$ & 20000000 & 3.327000 \\
\hline $\operatorname{Arco}(8,9)$ & $X$ & 1 & Infinita & 3.327000 \\
\hline $\operatorname{Arco}(9,10)$ & 0,063 & $\mathrm{X}$ & 15000000 & 3.327000 \\
\hline $\operatorname{Arco}(10,11)$ & $X$ & 1 & Infinita & 3.327000 \\
\hline
\end{tabular}

${ }^{6}$ Fonte: elaboração própria. 


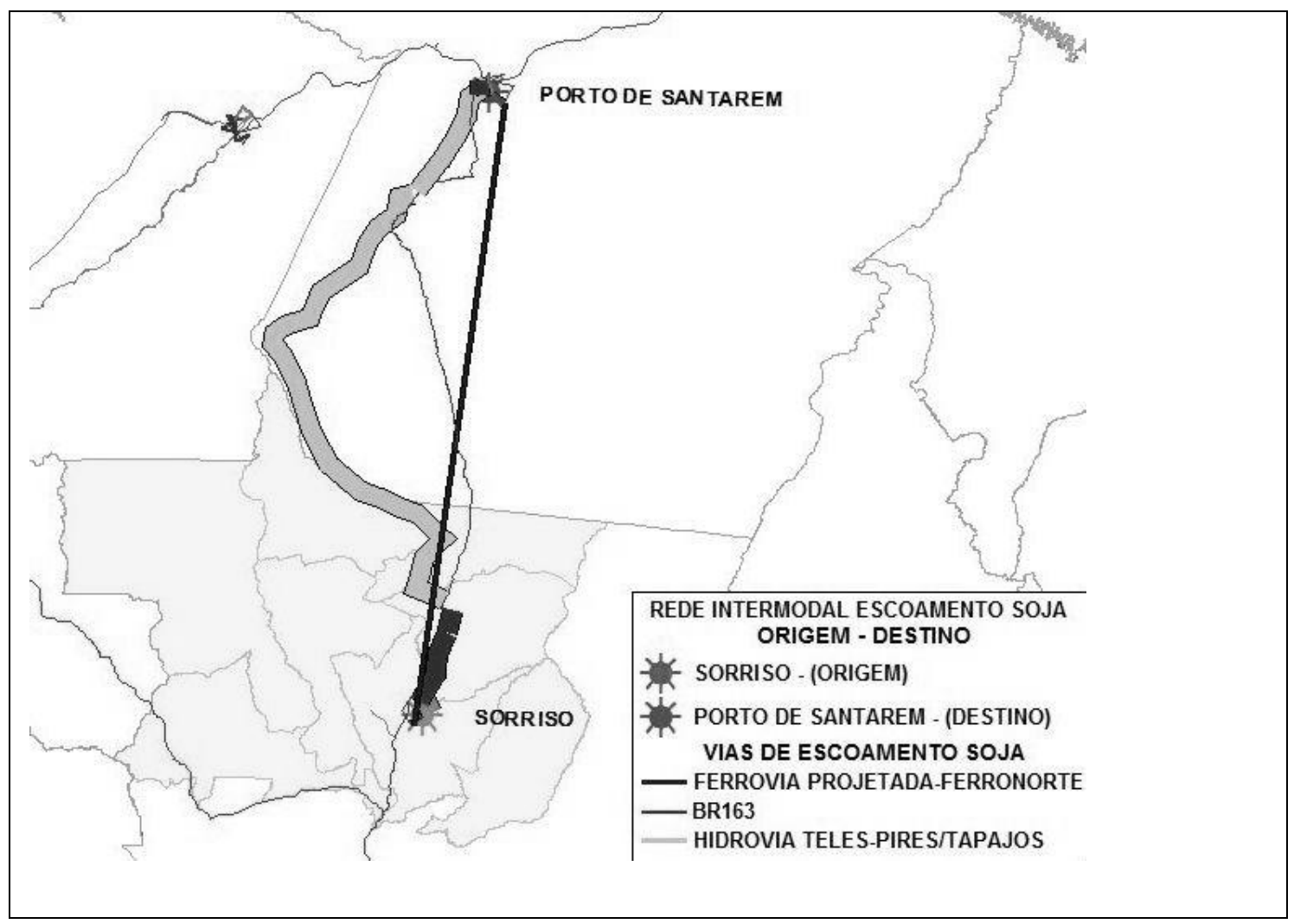

Figura 3 - Fluxos alocados na rede ${ }^{7}$

\section{Análise dos resultados}

Constata-se por meio dos resultados apresentados na Figura 3, que foram obtidos pelos critérios do algoritmo de fluxo a custo mínimo, um montante de 12 milhões de t/ano de soja foram alocados na hidrovia Tapajós/Teles-Pires, esgotando sua capacidade de movimentação no horizonte considerado. O restante, 3,3 milhões de toneladas, foi destinado para a ferrovia, não chegando a esgotar a capacidade de 20 milhões de t/ano desta. Nada foi alocado na rodovia BR-163, cuja capacidade ponderada no arco da rede foi de 15 milhões de t/ano.

A alocação máxima de fluxo de soja para a hidrovia Tapajós/Teles-Pires já era esperada diante do menor custo operacional $(\mathrm{R} \$ / \mathrm{t} . \mathrm{km})$ desta alternativa. Embora essa via apresente uma maior extensão com relação às demais alternativas, o seu custo operacional correspondente a $56 \%$ do custo da ferrovia e $35 \%$ com relação à rodovia.

\footnotetext{
${ }^{7}$ Fonte: elaboração própria.
} 
Por meio dos resultados obtidos, é possível verificar que a oferta de transporte existente e planejada de Sorriso até o porto de Santarém é capaz de atender toda a demanda de escoamento da soja produzida em Mato Grosso na data futura de 2023, apresentando um potencial de escoamento da produção para o norte, sem necessitar da utilização do porto de Santos que têm a desvantagem de apresentar um maior custo de transporte, uma vez que em sua maioria este é realizado pelo transporte rodoviário (Correa e Ramos, 2010). Com isso, é possível vislumbrar a necessidade e a oportunidade de maiores investimentos nessa rede logística no intuito de eliminar as deficiências que restringem a sua eficácia, notadamente nos terminais de transbordo e, principalmente, no porto de Santarém que teria que ser planejado em compatibilidade com as demandas potencial e sazonal alocadas na rede de oferta de transportes proposta.

Os resultados mostram que toda a alocação da demanda foi destinada para a hidrovia e ferrovia, deixando ociosa a BR-163, que é justamente a via prioritária para construção pelo Governo Federal.

Diante do realce da importância do ramal ferroviário no corredor norte, poder-se-ia pensar até na construção da ferrovia aproveitando o leito da BR-163, pois assim muitas questões ambientais e sociais que se constituem no principal problema para construção do ramal da Ferronorte estariam resolvidas, como, por exemplo, a eliminação da passagem do seu traçado por terras indígenas no Pará, além de aproveitar a faixa implantada e consolidada desde 1973, reduzindo o desmatamento, item crucial na tomada de decisão em qualquer obra na Amazônia. Haveria, ainda, condição de aproveitar de parte do estrato estrutural existente, reduzindo a movimentação de terra com reflexos positivos na redução do custo inicial do investimento. Esta poderia ser uma alternativa a ser estudada na diretriz escolhida no estudo de pré-viabilidade da obra.

Uma questão primordial quanto ao efetivo aproveitamento no caso da ferrovia planejada (Ferronorte) seria a falta de alocação para as cargas de retorno em volumes expressivos, aumentando a sua viabilidade financeira e econômica, promovendo redução significativa dos custos operacionais e, consequentemente dos fretes.

Para tanto, é necessário que haja um equilíbrio no fluxo dos produtos das regiões atendidas para que não ocorra ociosidade nos vagões de retorno, garantindo eficiência operacional na 
rede de transporte considerada. Isto poderá ser feito aproveitando a viagem de retorno para o transporte de insumos da agricultura como fertilizantes, calcário, combustíveis etc.

É necessário pensar que, para o horizonte estudado (2023), a implantação da ferrovia projetada e da hidrovia Tapajós/Teles-Pires é essencial para a otimização da rede de transporte considerada, propondo o deslocamento do vetor logístico para o norte como anteriormente mencionado, focando a intermodalidade como fator determinante para elevar a oferta de transporte e a competitividade do produto no mercado mundial com maior eficiência ambiental, social e econômica.

\section{Conclusão}

Em uma visão ampla, o procedimento proposto na Figura 1, considerado como objetivo adicional apresenta-se como uma ferramenta de apoio ao planejamento estratégico do transporte de carga na medida em que considera de forma estruturada a identificação dos vetores logísticos a serem considerados e a análise de oferta de transporte, como parte do diagnóstico da região de estudo em etapas iniciais de um modelo de planejamento de transportes de carga. Adicionalmente, considera-se a geração de viagens, escolha modal e alocação de fluxo em redes de transporte, etapas componentes do modelo de análise e previsão de demanda de viagens de 4 etapas, elemento central dos modelos de planejamento de transporte (Souza e D’Agosto, 2013).

Estando inserido em modelagem de maior abrangência, como destacado no parágrafo anterior, é possível considerar que o procedimento proposto teria aplicação para diferentes abrangências geográficas (de municípios a países), para diferentes tipos de carga (de carga geral com baixos volumes e altos valores agregados até granéis com elevados volumes e baixos valores agregados), considerando o fluxo em unidades de carga ou de veículos e considerando todos os modos de transporte ou suas combinações.

Dentro deste contexto, uma das aplicações considera a escolha de opções para o escoamento de produtos em corredores de exportação, que a exemplo da soja, pode ser ampliado para o transporte de toda uma seleção de cargas sólidas e líquidas, como commodities agrícolas, 
combustíveis, insumos de produção (siderurgia, metalurgia, indústria do cimento, agropecuária entre outras).

De modo a atender o objetivo principal deste trabalho, foi estabelecida uma alocação ótima de fluxo de carga em rede futura de oferta de transporte prevista pelo Plano Nacional de Logística e Transporte (PNLT), para o horizonte de 2023, para o escoamento de soja do norte do estado do Mato Grosso para o porto de Santarém.

Para tanto foi elaborado um procedimento, que guarda alguma analogia com o modelo de análise e previsão de demanda de quatro etapas, apresentado no item 2 do artigo. Por meio de sua aplicação foi possível verificar que o Porto de Santarém pode ser considerado uma alternativa para a exportação de soja oriunda de Mato Grosso, visto que a oferta de transporte existente e planejada de Sorriso até o porto de Santarém tem condições de atender a demanda de soja prevista para 2023, e que ainda apresenta um custo de transporte menor do que a alternativa praticada atualmente (utilização majoritária do porto de Santos), podendo oferecer maior competitividade do produto mato-grossense no mercado mundial.

Adicionalmente, a alternativa de exportação de soja oriunda de Mato Grosso pelo porto de Santarém pode priorizar o transporte intermodal rodo-hidroviário, contribuindo para melhor distribuição modal do transporte de carga no Brasil, um dos objetivos do PNLT. Além disso, seria feito por um corredor de escoamento que apresenta menor concorrência com outras cargas, tanto para o modo hidroviário quanto para o modo ferroviário, que o corredor de exportação atualmente utilizado.

Destaca-se que as alternativas definidas como as mais adequadas pelo modelo priorizam o uso dos modos hidroviário e o ferroviário, e que o modo rodoviário, apesar a alternativa priorizada atualmente para investimentos, ficou ocioso. Este resultado parece sinalizar que as diretrizes de planejamento estratégico para o transporte de carga relacionadas a este caso deveriam ser reavaliadas, tendo em vista que esforços e recursos podem estar sendo direcionados para a alternativa de transporte menos adequada.

Como limitações deste estudo, destaca-se que os resultados apresentados refletem a aplicação realizada e podem não representar a realidade para outras situações. Um dos principais aspectos neste sentido diz respeito à alocação de todo fluxo de carga na hidrovia 
Tapajós/Tele-Pires que considera que não haverá concorrência com outras cargas. Numa situação mais próxima da realidade é provável que parte da capacidade da hidrovia deva ser destinada para outras cargas e um maior uso da ferrovia seja verificado.

Outra limitação, que pode se refletir num aprimoramento para trabalhos futuros, diz respeito a não consideração dos tempos de transbordo. Além disso, os custos de transbordo também merecem um melhor detalhamento.

Como trabalhos futuros, sugere-se que sejam considerados também aspectos ambientais, tais como consumo de energia e emissões, para verificação da alternativa de maior ecoeficiência.

\section{Referências}

Associação das Hidrovias da Amazônia Oriental - AHIMOR (2012) Hidrovia Tapajós/Teles-Pires. Disponível em www.antaq.gov.br.

Barat, J. (1969) O investimento em transporte como fator de desenvolvimento regional: uma análise da expansão rodoviária no Brasil, Revista Brasileira de Economia, vol. 23, n. 3, pp. 25-52.

Branco, A. M. e Martins, M. H. (2006) Navegação fluvial no estado de São Paulo, São Paulo: Engenho.

Busacker, R. G. e Gowen, P. J. (1961) A procedure for determining a family of minimal-cost network flow patterns. O.R.O. Tech. Paper n. 15 - Johns Hopkins University.

Caliper Corporation (1996) Routing and logistics with TransCad, version 3.0. USA.

Campos, V. B. G. (1997) Método de alocação de fluxo no planejamento de transportes em situações de emergência: definição de rotas disjuntas. Tese (Doutorado) - Universidade Federal do Rio de Janeiro, Rio de Janeiro.

Companhia Nacional de Abastecimento - Conab (2012) Dados sobre a produção de soja no Brasil. Disponível em www.conab.gov.br.

Correa, V. H. C. e Ramos, P. (2010) Precariedade do transporte rodoviário brasileiro para o escoamento da produção de soja do centro-oeste: situação e perspectivas. Revista Economia e Sociologia Rural - RESR, vol. 48, n. 2, pp. 447-472.

CP Empreendimentos (2007) Ferronorte - análise da pré-viabilidade econômica do ramal CuiabáSantarém, Brasília-DF.

Departamento Nacional de Infraestrutura de Transporte - DNIT (2010) Informação sobre rodovia BR163. Disponível em www.dnit.gov.br.

Departamento Nacional de Infraestrutura de Transporte/Instituto Militar de Engenharia - DNIT/IME (2005) Estudo de viabilidade técnico-econômica da BR-163, Rio de Janeiro - RJ.

Fernández, A. J. C. (2004) Do cerrado à Amazônia: as estratégias sociais da economia da soja em Mato Grosso. Tese (Doutorado em Desenvolvimento Rural) - Universidade Federal do Rio Grande do Sul, Rio Grande do Sul. 
Fialho. F. (2010) O transporte hidroviário como solução logística e ambiental. Disponível em www.antaq.gov.br.

Hijjar, M. F (2004) Logística, soja e comércio internacional, ILLOS (Instituto de Logística e Suppy Chain), Rio de Janeiro-RJ.

Lavorentte, G. B. (2011) Caracterização das vias de exportação de soja do estado do Mato Grosso. Grupo de pesquisa e extensão em logística agroindustrial. Trabalho de Iniciação Científica ESALQ-LOG, Universidade De São Paulo.

Lemes, D. C. S. S. (2005) Geração e análises do cenário futuro como um instrumento do planejamento urbano e de transportes. Dissertação (Mestrado) - Universidade Federal de Uberlândia, Uberlândia.

Magalhães, S. L. M. (2008) Planejamento de transporte de um aglomerado urbano com intermodalidade por meio de transportes integrados. Tese (Doutorado em Engenharia de Transportes) - Universidade Federal do Rio de Janeiro, Rio de Janeiro.

Marrano, F. (2009) BR-163 - Solução logística e perspectivas de integração nacional. Disponível em www.fiemt.com.br.

Ministério dos Transportes (2007) Plano nacional de logística e transporte. Disponível em www.transportes.gov.br.

Morales, P. R. (1993) Modelo para gerenciamento de um corredor ferroviário de transporte de carga. Dissertação (Mestrado) - Instituto Militar de Engenharia, Rio de Janeiro- RJ.

Morlok, E. K. (1978) Introduction to transportation engineering and planning. N.York: McGraw-Hill.

Souza, C. D. R e D’Agosto, M. A. (2013) Modelo de quatro etapas aplicado ao planejamento de transporte de carga. Journal of Transport Literature, vol 7, n. 2.

Tedesco, G. M. I. (2008) Metodologia para elaboração do diagnóstico de um sistema de transporte. Dissertação (Mestrado) - Universidade de Brasília, Brasília. 